\title{
Quasars resolved?
}

\section{from M.G. Edmunds}

SOME controversies seem to go on for ever, but the quasar redshift debate must surely soon come to an end. During the past year the arguments for interpreting the redshifts as an indicator of large distances to the quasars have grown stronger, despite a spirited rearguard action by the main champion of a non-cosmological interpretation.

In a recent paper in the Astrophysical Journal ${ }^{1}$, Arp reports the discovery of several new quasars which lie in directions close to galaxies not very far away in space from our own Galaxy. Arp has maintained that there is evidence for a considerable excess in the number of quasars per unit area of sky around nearby galaxies (as seen from Earth), compared with regions away from obvious galaxies. He has suggested that this supports the idea that quasars are objects ejected by the galaxies, since the conventional 'cosmological' interpretation of redshifts would require an approximately random distribution of quasars on the sky. Arp's latest claim is that around a sample of galaxies which are companions to large nearby spiral galaxies, the observed density of quasars is so high that the probability of the configurations occurring by a chance fluctuation in a random distribution would be an astounding $10^{-17}$. If this statistic were right, a cosmological interpretation would be very hard to justify but the estimate does not stand up to closer examination.

Arp has performed a useful survey in looking at some 34 areas of sky and, although it is unfortunate that he does not give details of the exact area searched in each case, we may use his results to discuss the statistical significance of association. His analysis is to assign a probability of occurrence individually to each association he finds, and then combine these together to obtain an overall likelihood. A rather simpler (and probably preferable) approach is to ask what the expected number of associations would be, given a random distribution and a search of the 34 areas, and compare this with the observed numbers. In his analysed sample Arp finds 13 quasars at an angle of less than 300 arc s from the 34 companion galaxies, and all the quasars are brighter than about 20th magnitude on his magnitude scale. If we assume the search for these objects is complete out to at least $300 \mathrm{~s}$, then if there are on average $n$ quasars per square degree the expected number within $300 \mathrm{~s}$ of any one galaxy will be of order

$$
\pi \times\left(\frac{300}{60 \times 60}\right)^{2} \times n,
$$

and 34 times this for the total of 34 galaxies sampled. The total predicted is thus $0.74 n$. The problem comes in the choice of $n$.

Arp initially assumes 10 per square degree down to 20th magnitude, giving a predicted number of 7 against an observed 13, which is perhaps a larger fluctuation than could reasonably be ascribed to chance (although not a fluctuation with as small a chance as $10^{-17}$ ). But it is not obvious that 10 is the correct value to take for $n$. By assuming instead 18 per square degree down to 20th magnitude observed and predicted values can be made to agree, and as emphasized by Véron and Véron ${ }^{2}$, a reconsideration of all available data on quasar densities shows that such an assumption is not unreasonable. Osmer ${ }^{3}$ found 14 per square degree down to 19.75 magnitude. Véron and Véron suggest that the major problem in the comparison of the various estimates of surface density is the variation of magnitude scales between different observers. Since the observed numbers are a very sensitive function of limiting magnitude, a difference between magnitude estimates propagates very strongly into a difference in surface density. A problem with Arp's statistics is emphasized when he claims that even if $n$ were as high as 100 , the probability of the associations he observed occuring by change would still be only $10^{-5}$, and we note that in this case he should have discovered about 74 quasars within 300 arc s of the galaxies - and indeed the chances of discovering just 13 are very small, but the result would then imply anti-association!

These kinds of statistical argument cannot be considered satisfactory until reasonably large areas of sky have been surveyed completely to a consistent magnitude limit. Arp could fairly argue that most of the quasars he finds are substantially brighter than 20th magnitude (by up to 0.5 magnitude), but again the difficulty arises over variation of magnitude scales - an exactly similar search in 34 areas not centred on companion galaxies would be a useful control sample in setting the relevant (inevitably observer-dependent) value of $n$. Further, neither Arp nor this discussion have taken account of possible enhancement of the surface density of quasars by the brightening of distant quasars by the gravitational lens if the galaxies have massive haloes of stellar mass objects ${ }^{4}$.

Strong evidence that most quasars are at the cosmological distances implied by their redshifts has come from the work of two groups, one Canadian ${ }^{5}$, the other from the United States and Germany ${ }^{6}$. By definition quasars are 'quasi-stellar' and hence have

\section{Erratum: The Flavells}

The article The mystery of the mouse a-globin pseudogene (Nature 295, 370; 1982) was written by Andrew Flavell of the Imperial Cancer Research Fund Laboratories, Mill Hill, London. We regret that we erroneously attributed authorship to his brother R.A. Flavell of the National Institute of Medical Research, Mill Hill, London. unresolved circular images on direct photographic plates, although some spatial structure has been recognized in a few cases. What these groups have done is to look carefully at the images of some two dozen low redshift (that is, not too distant) quasars, including the classic 3C273. By scanning both quasars and star images with an accurate measuring machine, they have been able to 'resolve' the quasars by demonstrating that in nearly every case the images of the quasars are more extended than those of stars. The light distribution of these extended images is consistent with their being galaxies - although the data cannot reliably distinguish between the light distribution of a spiral and an elliptical galaxy. Furthermore, the apparent size of the extended image decreases with increasing redshift of the quasar in just the way expected if the underlying galaxies were all of comparable size and the redshift were an indicator of distance.

These results support the idea that what we see as quasars are just the very bright active nuclei of some galaxies. This is not to say that the underlying galaxies are completely normal - the intense radiation source at the centre undoubtedly has some influence on the morphology and evolution of the system. A few of the quasar images are extended into huge jets (as in the case of 3C273) of comparable size to the underlying galaxy - a phenomenon not seen on anything like this scale in normal galaxies - and for a few quasars, the nebulosity around the centre has previously been shown to have the characteristics of highly excited gas.

It must be conceded to Arp that it remains possible that a subset of quasars could have a non-cosmological component to their redshift, although the apparent similarity of nearly all quasar spectra might then be surprising. Apparent alignments of quasar groups on the sky could be interpreted as evidence for physical association of quasars of very different redshifts, and this would be impossible if the redshifts were true distance indicators. However, the statistical simulations of Zuiderwijk ${ }^{7}$ confirm previous suggestions that such alignments as are observed are quite likely to arise by chance juxtaposition on the sky, with no physical association.

It was the very considerable spread in the intrinsic luminosity of quasars which vitiated the plotting of a simple magnituderedshift Hubble diagram that would long ago have settled doubts about their distance. The new information of angular diameter versus redshift of Wyckoff et al. must, however, considerably strengthen the cosmological distance interpretation of quasar redshifts.

M. G. Edmunds is in the Department of Applied Mathematics and Astronomy, University College, PO Box 78, Cardiff CFI IXL. 